\title{
Distinct metabolic features in the plasma of patients with silicosis and dust-exposed workers in China: a case-control study
}

\author{
Changjiang Xue, Na Wu, Yali Fan, Jing Ma and Qiao Ye*
}

\begin{abstract}
Background: Silicosis is a progressive pneumoconiosis characterized by interstitial fibrosis following exposure to silica dust. The role of metabolic dysregulation in the pathogenesis of silicosis has not been investigated in detail. This study aimed to identify different metabolic features in the plasma of patients with silicosis and dust-exposed workers without silicosis in metabolomics studies.

Methods: Patients with silicosis, dust-exposed workers (DEWs) without silicosis and age-matched healthy controls were recruited in a case-control study. The metabolomics analyses by ultra-high performance liquid chromatography-mass spectrometry were conducted. Distinct metabolic features (DMFs) were identified in the pilot study and were validated in the validation study. The enriched signalling pathways of these DMFs were determined. The ability of DMFs to discriminate among the groups was analysed through receiver operating characteristic (ROC) curves. The correlations between DMFs and clinical features were also explored.
\end{abstract}

Results: Twenty-nine DMFs and 9 DMFs were detected and had the same trend in the pilot study and the validation study in the plasma of the DEW and silicosis groups, respectively. Sphingolipid metabolism was the major metabolic pathway in the DEWs, and arginine and proline metabolism was associated with silicosis. Twenty DMFs in the DEWs and 3 DMFs in the patients with silicosis showed a discriminatory ability with ROC curve analysis. The abundance of kynurenine was higher in Stage III silicosis than in Stage I or Stage II silicosis. L-arginine and kynurenine were both negatively correlated with the percentage of forced vital capacity predicted in silicosis.

Conclusions: Distinct metabolic features in the plasma of DEWs and the patients with silicosis were found to be different. Sphingolipid metabolism and arginine and proline metabolism were identified as the major metabolic pathway in the DEW and silicosis groups, respectively. L-arginine and kynurenine were correlated with the severity of silicosis.

Keywords: Silicosis, Plasma, Metabolomics, Pulmonary function, L-arginine, Kynurenine

\section{Background}

Silicosis is a progressive pneumoconiosis characterized by nodular interstitial fibrosis following exposure

\footnotetext{
*Correspondence: yeqiao_chaoyang@sina.com

Department of Occupational Medicine and Toxicology, Clinical Centre for Interstitial Lung Diseases, Beijing Chao-Yang Hospital, Capital Medical University, No. 8 Workers' Stadium South Road, Chao-Yang District, Beijing 100020, China
}

to silica dust [1]. Disease progression of silicosis usually leads to irreversible complications and death. As observed in a recent American Thoracic Society review, workplace exposures contribute substantially to the burden of chronic respiratory diseases such as silicosis [2]. China, where industries with silica exposure have not been strongly regulated and poor personal protection due to insufficient attention, has 
exhibited a sustained epidemic of silicosis [3]. According to a report from China's Ministry of Health, more than 23 million workers are exposed to crystalline silica in China [4]. Currently, the clinical diagnosis and monitoring of silicosis mainly relies on a history of occupational exposure and radiological abnormalities [5].

Crystalline silica entering the airways is engulfed by macrophages, causing necrosis of the phagocytes, and then, the internalized silica is released again and engulfed by other macrophages. The repeated process of phagocytosis, necrosis and rephagocytosis of the cells induces inflammation and activation of the reactive oxygen species system, which is associated with pulmonary interstitial fibrosis [6-8]. Proteomic analysis has suggested that some proteins have been found to be closely related to the occurrence and development of silicosis $[9,10]$. Most proteins were enriched in immune system processes, oxygen transporter activity, phagosome, lysosome and extracellular matrix (ECM)-receptor interactions [11]. Our previous study showed that serum Krebs von den Lungen 6, surfactant protein $\mathrm{D}$ and matrix metalloproteinase- 2 were potential biomarkers for diagnosing and monitoring silicosis [12]. Furthermore, many metabolites are involved in the pathogenesis of silicosis and may play a predictive role in the diagnosis and severity of the disease. Research has also suggested that cholesterol oxidation can be used as an important marker and lipid metabolism is affected and oxidative lipid damage is triggered in silicosis [13].

Metabolomics, which is a systematic investigation of all metabolic responses in a biological system, provides a comprehensive and quantitative method of studying a complete set of intracellular and extracellular metabolites [14, 15]. Metabolomics studies have helped elucidate the pathogenesis and identify potential biomarkers for the diagnosis or prognosis of pulmonary fibrosis $[16,17]$. Metabolic pathway changes in sphingolipid metabolism, the arginine pathway, glycolysis, mitochondrial beta-oxidation and the tricarboxylic acid cycle were found in the lung tissues of patients with idiopathic pulmonary fibrosis (IPF) in a metabolomics study [18]. Silicosis probably has similar pathogenesis as IPF, which may be related to the pathological manifestation of the production of collagen fibres and pulmonary interstitial fibrosis [19]. The role of metabolic dysregulation in the pathogenesis of silicosis has not been investigated in detail.

To identify the distinct metabolic features (DMFs) in silicosis, we evaluated the metabolic profiles in the plasma of patients with silicosis compared with dust-exposed workers without silicosis and healthy controls using pilot and validation metabolomics studies.

\section{Methods \\ Study design and population}

This case-control study adopted a cross-sectional design and recruited a total of 170 individuals. In the pilot phase, the individuals studied were 30 patients with silicosis, 30 dust-exposed workers (DEWs) without silicosis and 20 age-matched healthy controls. There were 30 individuals in each group in the validation phase. No individual participated in both the pilot and validation studies. All patients with silicosis were sequentially obtained from the Department of Occupational Medicine and Toxicology, Beijing Chao-Yang Hospital, during a more than 2-year period (January 2018 to December 2019). They were diagnosed according to the radiological criteria of pneumoconiosis based on the 2011 International Labour Organization (ILO) classification [20]. Patients with chronic obstructive pulmonary disease, asthma, tuberculosis, autoimmune disease, uncontrolled hypertension and diabetes, severe liver and kidney dysfunction, malignant tumours, and patients who abuse alcohol were excluded. The DEWs had occupational exposure to silica dust and underwent all examinations without showing evidence of silicosis. The controls comprised 50 agematched healthy volunteers from the health examination centre of Beijing Chao-yang Hospital during the same period of time.

Clinical data were retrieved from medical reports and included age, sex, height, weight, smoking status, occupational history, current and past medical history and family history. The occupational history (including type of exposure and start and end dates of employment) was collected, and all jobs within the working life were taken into account. The subjects with silicosis and the DEWs enrolled in the study were local residents who had been exposed to silica dust during excavation and digging (83, $69.2 \%)$, polishing and buffing $(16,13.3 \%)$, handling raw materials $(14,11.7 \%)$ and rock blasting and sand blasting $(7,5.8 \%)$. Our hospital is a regional medical centre for occupational diseases. The smoking status of all individuals was carefully determined and categorized as nonsmokers, ex-smokers (had quit smoking $\geq 12$ months previously), and smokers (currently smoking or had quit smoking $<12$ months previously). All individuals underwent chest X-ray/chest high-resolution computed tomography and pulmonary function tests (see Additional file 1). 


\section{Sample preparation}

Briefly, the plasma samples of all subjects were collected on an empty stomach in the morning both in the pilot study and the validation study and stored at $-80^{\circ} \mathrm{C}$ until metabolic analysis. All samples for the two studies were handled according to the same measurement procedure. Frozen plasma was gently thawed at $4{ }^{\circ} \mathrm{C}$. Then, $100 \mu \mathrm{L}$ of plasma was added to $300 \mu \mathrm{L}$ of methanol or acetonitrile, thoroughly mixed on a vortex mixer for $15 \mathrm{~s}$ three times, and centrifuged at 12,000 rpm for $5 \mathrm{~min}$. Then, $100 \mu \mathrm{L}$ of supernatant was pipetted into vials to be analysed on an ultra-high-performance liquid chromatography-mass spectrometry (UHPLC-MS) instrument for UHPLC-MS analysis.

\section{Metabolomics profiling by UHPLC-MS}

The workflow of our work was shown in Fig. 1. The samples were separated by reversed-phase chromatography and hydrophilic chromatography. In this study, the plasma samples were randomly numbered for metabolite extraction and analytical run. All samples were processed and detected continuously within 2 days. Pooled quality control samples were prepared by mixing all of the samples to ensure the data quality of metabolic profiling (see Additional file 1: Figure S1).

For reversed-phase chromatography on a $\mathrm{C}_{18}$ column, the plasma samples were melted, chloroform/methanol

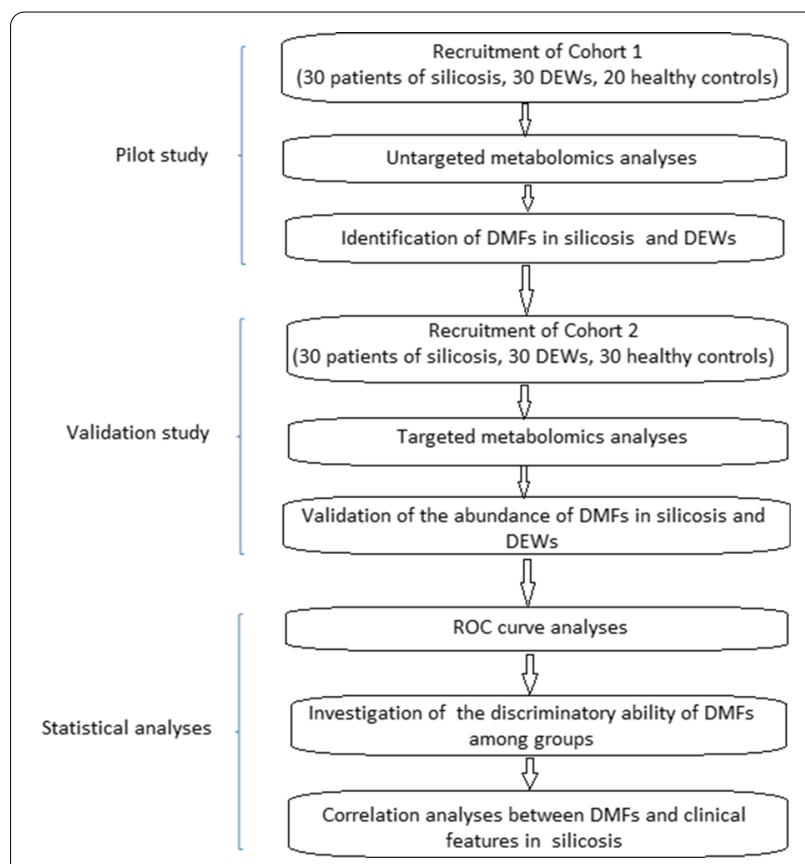

Fig. 1 The workflow of our work. The pilot study phase (80 individuals), validation study phase (90 individuals) and statistical analyses were incorporated into our study was added, ultrasonication was conducted, and water was then added to the mixture. After centrifugation, the lower chloroform was concentrated and dried; then, isopropyl alcohol/acetonitrile was added and dissolved by ultrasonication. The solution was centrifuged, and the supernatant was transferred for analysis. Unlike the reversed-phase chromatography, in which hydrophilic chromatographic analysis was conducted on a HILIC column, acetonitrile was added to the melted plasma samples, the mixture fully oscillated, and the upper solution was taken to be tested. The pooled quality-controlled sample was tested at the beginning of the test. Then, every seven samples were tested again to monitor the process to ensure the reliability of the data. A hybrid quadrupole-orbitrap mass spectrometer (Q Exactive, Thermo Fisher Scientific, Beijing, China) equipped with a HESI-II probe was used for mass spectrometry (MS). The liquid quality system was controlled by Xcalibur 2.2 SP1.48 software. Data acquisition and quantitative treatment of targeted metabolites were performed using the same software.

\section{Statistical analysis}

The UHPLC-MS raw data were processed using Progenesis QI (Waters Corporation, Milford, MA, USA), which performed run alignment, peak picking, adduct deconvolution and feature identification against the HMDB database and laboratory-specific reference database. The fragmentation mass spectrum of metabolites was matched to fragmentation mass spectra in library and scored. The results with higher score were accepted as the identification results. Principal component analysis (PCA) and orthogonal partial least-squares-discriminant analysis (OPLS-DA) were performed using Simca (Umetrics Version 14.1).The corresponding parameters of models including $R^{2} X, R^{2} Y$, and $Q^{2}$ were obtained and used to ensure the quality of the multivariate models and to avoid the risk of overfitting. $t$ tests and variable importance for projection (VIP) statistics were used, and metabolites with significant change were filtered out based on VIP values (VIP $>1)$ and $t$ tests $(P<0.05)$. MetaboAnalyst 4.0 software was used for pathway enrichment analysis. The Kyoto Encyclopedia of Genes and Genomes (KEGG) is a self-sufficient, integrated resource consisting of genomic, chemical and network information.

The validation data were processed using Skyline (MacCoss Lab). Compared with the peak intensity of the standard reference, the concentration of metabolites was calculated and statistical analyses were performed with SPSS version 22.0 (IBM Corp., Armonk, NY, USA). Data are expressed as mean \pm standard deviation. Group differences were examined using $t$ tests and one-way analysis of variance for continuous variables, chi-square test 
for count data and the Kruskal-Wallis test for non-normally distributed median values. Correlations between parameters were assessed by Pearson's correlation coefficient. The levels of the DMFs were further analysed by a receiver operating characteristic (ROC) curve to determine the area under the curve (AUC) and the sensitivity and specificity. A $P$ value of $<0.05$ was considered statistically significant.

\section{Results}

\section{Demographics of the study population}

The demographics of each group in both the pilot and validation studies were summarized in Table 1 . There was no significant difference in sex, age, body mass index or smoking status among the three groups.

\section{PCA and OPLS-DA}

In this study, the differentially regulated plasma metabolites in the patients with silicosis, DEWs and healthy controls were searched by nontargeted metabolic profiling using UHPLC-MS, and PCA, using the unsupervised model, was performed to reveal the differences in the metabolic profiles of samples among the groups. The PCA score plot exhibited clear clusters of plasma samples among the silicosis, DEW and control groups both in the $C_{18}$ column $\left(R^{2} X=0.725, Q^{2}=0.472\right)$ and the HILIC column $\left(R^{2} X=0.554, Q^{2}=0.296\right)$, as shown in Fig. 2a, b. For further analysis of the metabolic differences between the controls and DEWs and between the controls and patients with silicosis, OPLS-DA, a supervised method for pattern recognition, was applied. As illustrated by the OPLS-DA score plot, the plasma samples in the DEW group were clearly separated from those in the control group both in the $C_{18}$ column $\left(R^{2} Y=0.973, Q^{2}=0.952\right)$ and the HILIC column $\left(R^{2} Y=0.983, Q^{2}=0.966\right)$ (Fig. 2c, d). Those in the silicosis group were clearly separated from those in the control group both in the $\mathrm{C}_{18}$ column $\left(R^{2} Y=0.926, Q^{2}=0.859\right)$ and the HILIC column $\left(R^{2} Y=0.962, Q^{2}=0.848\right)$ (Fig. 2e, f).

\section{Analysis of the metabolic pathways in the DEWs and patients with silicosis}

To identify the metabolic networks and the biological relevance of the identified DMFs in the DEW and silicosis groups, we used MetaboAnalyst 4.0 software and the KEGG database. Sphingolipid metabolism and arginine and proline metabolism were the major metabolic pathways in the DEW and silicosis groups, respectively (Fig. 3).

\section{Identification of the DMFs in the DEWs and patients with silicosis compared with the controls in the pilot study} Approximately 3336 metabolic features, including 1223 $\mathrm{C}_{18}$ positive features, $1069 \mathrm{C}_{18}$ negative features and 1044 HILIC features were found and used for the further statistical analysis. OPLS-DA score plots were used to identify the DMFs for distinguishing the DEWs and patients with silicosis from the controls. According to the cutoff of VIP $>1$ and $P<0.05$ based on OPLS-DA model analysis, 49 DMFs were detected in the plasma of the DEWs; 44 DMFs (21 lipids, 6 amino acids and 17 carnitines) increased and 5 DMFs (4 lipids and 1 amino acid) significantly decreased compared with the controls.

Table 1 Baseline demographics of the study population

\begin{tabular}{|c|c|c|c|c|c|c|c|c|}
\hline & \multicolumn{4}{|l|}{ Pilot phase } & \multicolumn{4}{|c|}{ Validation phase } \\
\hline & $\mathrm{SIL}$ & DEW & CON & $P$ value & SIL & DEW & CON & $P$ value \\
\hline $\mathrm{N}$ & 30 & 30 & 20 & & 30 & 30 & 30 & \\
\hline Male:Female & $25: 5$ & $27: 3$ & $14: 6$ & 0.148 & $27: 3$ & $27: 3$ & $24: 6$ & 0.095 \\
\hline Age (years) & $59.3 \pm 10.1$ & $63.0 \pm 4.7$ & $59.5 \pm 9.9$ & 0.182 & $56.2 \pm 12.9$ & $60.1 \pm 7.9$ & $55.1 \pm 11.1$ & 0.176 \\
\hline Smokers: non-smokers & $20: 10$ & $22: 8$ & $11: 9$ & 0.405 & $21: 9$ & $20: 10$ & $18: 12$ & 0.709 \\
\hline Current smokers:ex-smokers & $3: 17$ & $9: 13$ & $4: 7$ & 0.166 & $5: 16$ & $7: 13$ & $7: 11$ & 0.572 \\
\hline $\mathrm{BMI}\left(\mathrm{kg} / \mathrm{m}^{2}\right)$ & $24.6 \pm 3.2$ & $23.7 \pm 2.3$ & $23.6 \pm 2.12$ & 0.345 & $22.8 \pm 3.3$ & $24.4 \pm 3.3$ & $23.8 \pm 3.2$ & 0.155 \\
\hline Duration of exposure (years) & $13.1 \pm 7.2$ & $9.3 \pm 3.3$ & NA & 0.002 & $11.8 \pm 6.5$ & $10.2 \pm 5.0$ & NA & 0.033 \\
\hline FVC (predicted \%) & $77.6 \pm 20.8$ & $82.9 \pm 22.3$ & $83.2 \pm 15.6$ & 0.047 & $75.2 \pm 23.3$ & $80.4 \pm 17.5$ & $82.8 \pm 17.4$ & 0.039 \\
\hline $\mathrm{FEV}_{1}($ predicted \%) & $76.2 \pm 19.7$ & $79.6 \pm 18.7$ & $80.4 \pm 12.1$ & 0.216 & $77.7 \pm 18.0$ & $79.1 \pm 19.6$ & $80.7 \pm 11.7$ & 0.054 \\
\hline $\mathrm{FEV}_{1} / \mathrm{FVC}(\%)$ & $81.1 \pm 20.4$ & $83.1 \pm 24.6$ & $82.8 \pm 18.3$ & 0.428 & $80.5 \pm 19.4$ & $82.0 \pm 20.0$ & $81.9 \pm 14.2$ & 0.366 \\
\hline $\mathrm{DL}_{\mathrm{CO}}($ predicted \%) & $76.7 \pm 17.2$ & $80.8 \pm 18.4$ & $81.2 \pm 10.5$ & 0.105 & $73.3 \pm 15.4$ & $79.4 \pm 16.3$ & $80.6 \pm 11.1$ & 0.233 \\
\hline
\end{tabular}

$P$ values were computed by chi-square test for sex and smoking status, one-way analysis of variance for age, $\mathrm{BMI}_{1} \mathrm{FVC}, \mathrm{FEV}{ }_{1}, \mathrm{FEV}{ }_{1} / \mathrm{FVC}$ ratio and $\mathrm{DL} \mathrm{CO}_{\mathrm{C}} t \mathrm{t}$ test was used to estimate duration of exposure

SIL patients with silicosis, DEW dust-exposed workers without silicosis, CON healthy control, BMI body mass index, FVC forced vital capacity, FEV forced expired volume in $1 \mathrm{~s}, D L_{C O}$ diffusing capacity of the lung for carbon monoxide, $N A$ not available 

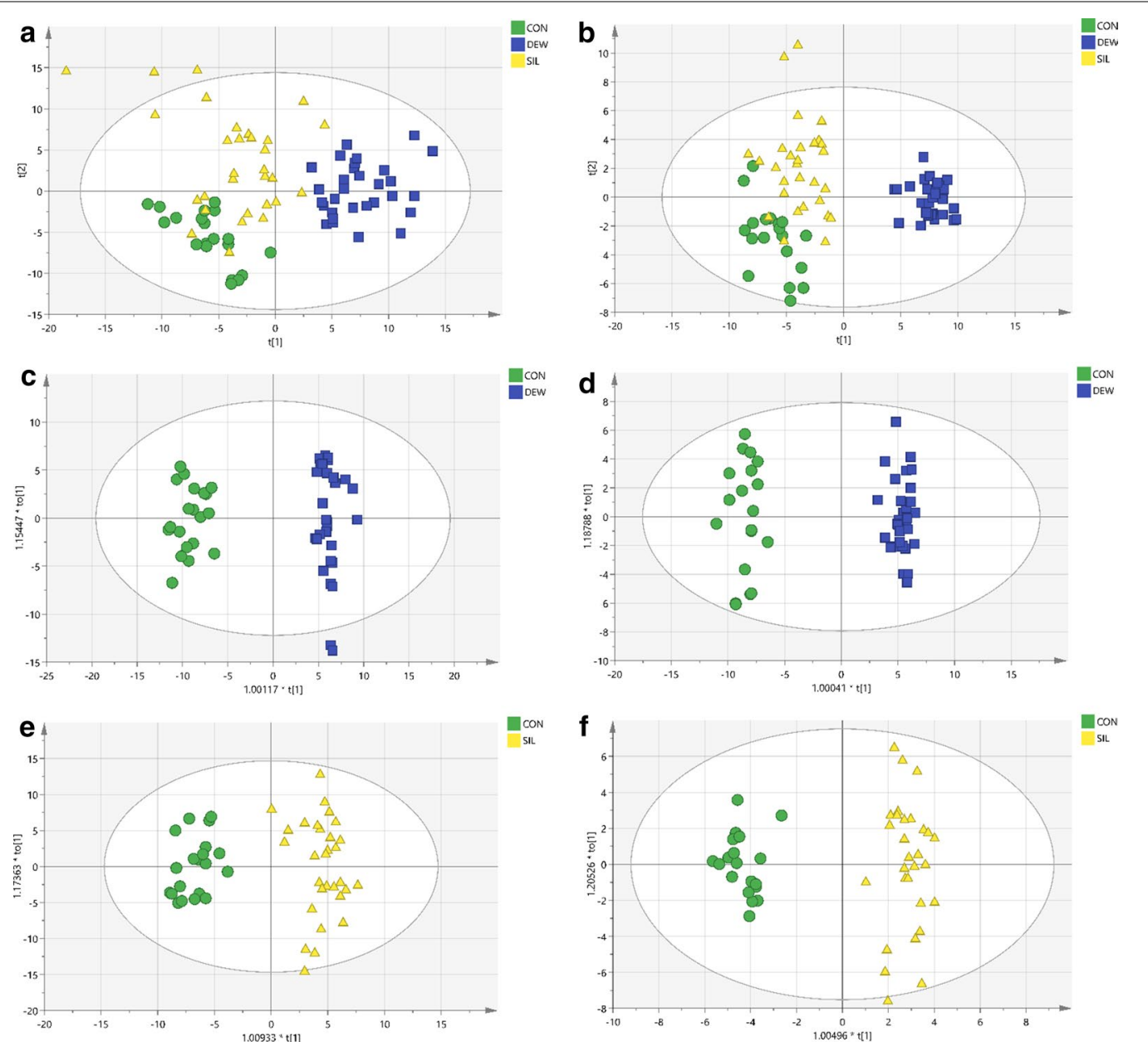

Fig. 2 PCA and OPLS-DA of the metabolic profiles of plasma samples in silicosis (SIL), dust-exposed workers (DEW) and healthy control (CON) groups. a PCA analysis in $C_{18}$ mode. b PCA analysis in HILIC mode. c OPLS-DA analysis in $C_{18}$ mode comparing DEW and CON. d OPLS-DA analysis in HILIC mode comparing DEW and CON. e OPLS-DA analysis in $\mathrm{C}_{18}$ mode comparing SIL and CON. $\mathrm{O}$ OPLS-DA analysis in HILIC mode comparing SIL and CON
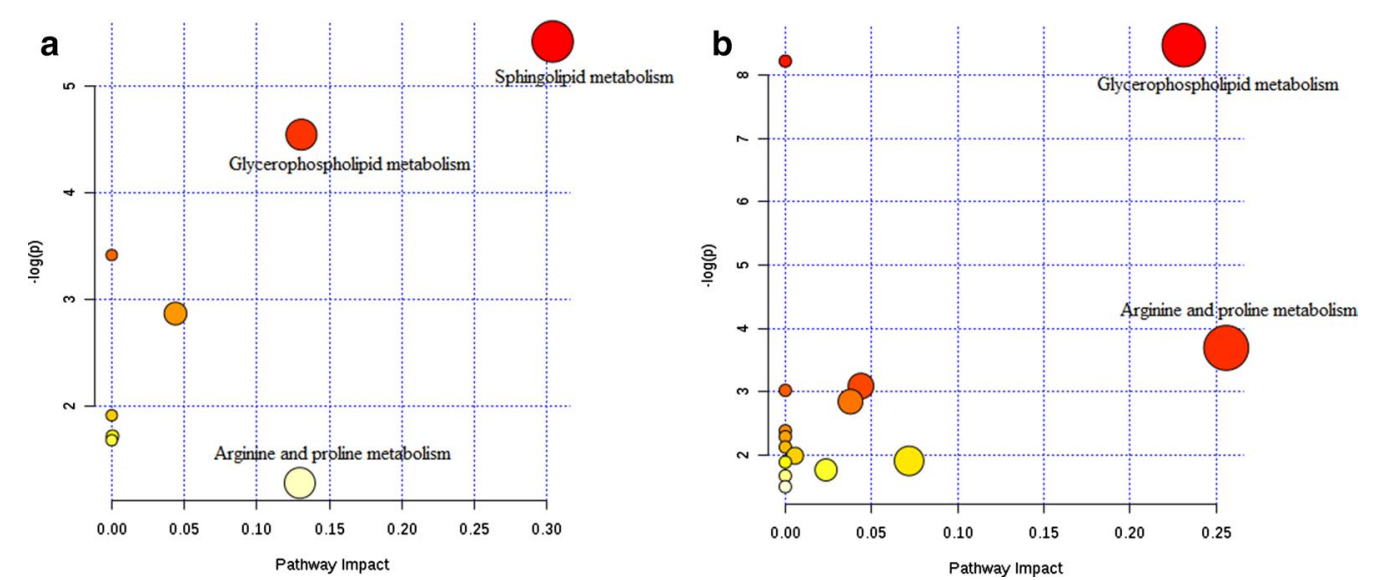

Fig. 3 Metabolic pathways in a dust-exposed workers without silicosis and $\mathbf{b}$ the patients with silicosis. Metabolomics view displays matched pathways as circles. The colour and size of each circle is based on the $P$ value and pathway impact value, respectively 
Thirty-seven DMFs were detected in the plasma of the patients with silicosis; 24 DMFs (16 lipids, 4 amino acids and 4 carnitines) increased and 13 DMFs (10 lipids and 3 amino acids) significantly decreased compared with the controls.

\section{Validation of the abundance of the identified DMFs in the validation study}

To validate the abundance of the DMFs, we performed targeted metabolomics analysis, and the difference in the abundance of the DMFs among the silicosis, DEW and healthy control groups was analysed in the validation study. The profiles of the DMFs revealed a clear difference between the DEWs and the controls and between the patients with silicosis and the controls in the form of heat maps (Additional file 1: Figure S2, S3).

The abundance of 29 identified DMFs in the DEWs and 9 identified DMFs in the patients with silicosis had the same trend in the pilot study and the validation study (Additional file 1: Table S1, S2). To assess the discriminatory ability of all the aforementioned DMFs between the controls and the DEWs and between the controls and the patients with silicosis, we performed ROC curve analyses to calculate the AUC. Twenty of 29 DMFs, mainly carnitines and lipids, had distinct values in the plasma samples of the DEWs compared to those of the controls (Additional file 1: Figure S4). In the plasma samples of the patients with silicosis compared with the controls, 3 amino acid DMFs, kynurenine, L-arginine and creatine, could discriminate between the groups. The abundances and the ROC curves are shown in Fig. 4. These three DMFs were identified and quantified by standard reference materials. MS/MS spectrum from standard reference, pooled plasma sample from control group, silicosis group and all samples were compared, which supported the identification results of the metabolites (Additional file 1: Figure S5-S7).

\section{Correlations between DMFs and the severity of silicosis}

To explore the correlation between the three DMFs and the severity of silicosis, we classified all patients with silicosis into three stages according to the diagnostic criteria of pneumoconiosis based on the 2011 ILO classification. The abundance of kynurenine was higher in Stage III silicosis than in Stage I or Stage II silicosis. However, those of creatine and L-arginine were not significantly different among the various stages of silicosis (Table 2).

Pulmonary function was assessed for each patient with silicosis in the validation study. As shown in Fig. 5, the abundances of L-arginine and kynurenine were negatively correlated with the predicted percentage of forced vital capacity (FVC\% predicted) $(P<0.05)$ but not correlated with other lung function values in silicosis.

\section{Discussion}

Silicosis is a well-known fibrotic lung disease caused by prolonged inhalation of crystalline silica. In order to explore the metabolic mechanism of silicosis, we designed this study to compare the metabolic features of the patients of silicosis and DEWs in metabolomics studies. Twenty-nine DMFs were identified in the plasma of the DEWs in the pilot and validation study. Sphingolipid metabolism was the major metabolic pathway in the DEWs. It showed that the metabolic mechanism was decidedly altered once dusts entered into the respiratory tracts in the dust exposure environment. However in the patients with silicosis, 9 DMFs were detected to be consistent in the pilot and validation study. Arginine and proline metabolism was the major metabolic pathway in silicosis.

In the present study, 20 DMFs, mainly lipids and carnitines, could distinguish the DEWs from the healthy controls. Lipids are essential nutrients in humans and are the main components of cell membranes and cellular energy storage. Lipids are related to signal transduction, enzyme activation, growth factors and antioxidants, signal recognition and immunity [21-23]. Recently, the role of lipids has attracted increased attention in lungs and respiratory diseases, including cystic fibrosis, asthma and chronic obstructive pulmonary disease, which are all associated with abnormal metabolisms [24-26]. Researchers have revealed vital information regarding lipid metabolism in IPF patients, and more importantly, a few potentially promising biomarkers were first identified and may have a predictive role in monitoring and diagnosing IPF [18, 27, 28]. Lysophosphatidylcholine (LysoPC), an intermediate metabolite of sphingolipids, was shown to be a potential biomarker in the serum of patients with IPF by pilot and further validation studies using UHPLC-MS [29]. Carnitine is related to the metabolism of fat into energy in the body and can promote the transport and oxidation of fatty acids and the utilization of carbohydrates and amino acids, improve body tolerance, prevent lactic acid accumulation, and delay ageing and is involved in antioxidant activities [30]. Carnitine was significantly decreased in the lung tissue and reduced mitochondrial beta-oxidation in IPF [17]. In the early phase of silicosis, the enhancement of macrophage phagocytosis, apoptosis, oxidative stress and inflammation is caused by inhaled crystalline silica [31-33] and is closely related to the abnormal metabolism of lipids and carnitines. The data suggested that sphingolipid metabolism was the major metabolic pathway in the DEWs and that LysoPC and lysophosphatidylethanolamine (LysoPE), the intermediate metabolites of sphingolipid metabolism, were upregulated. 

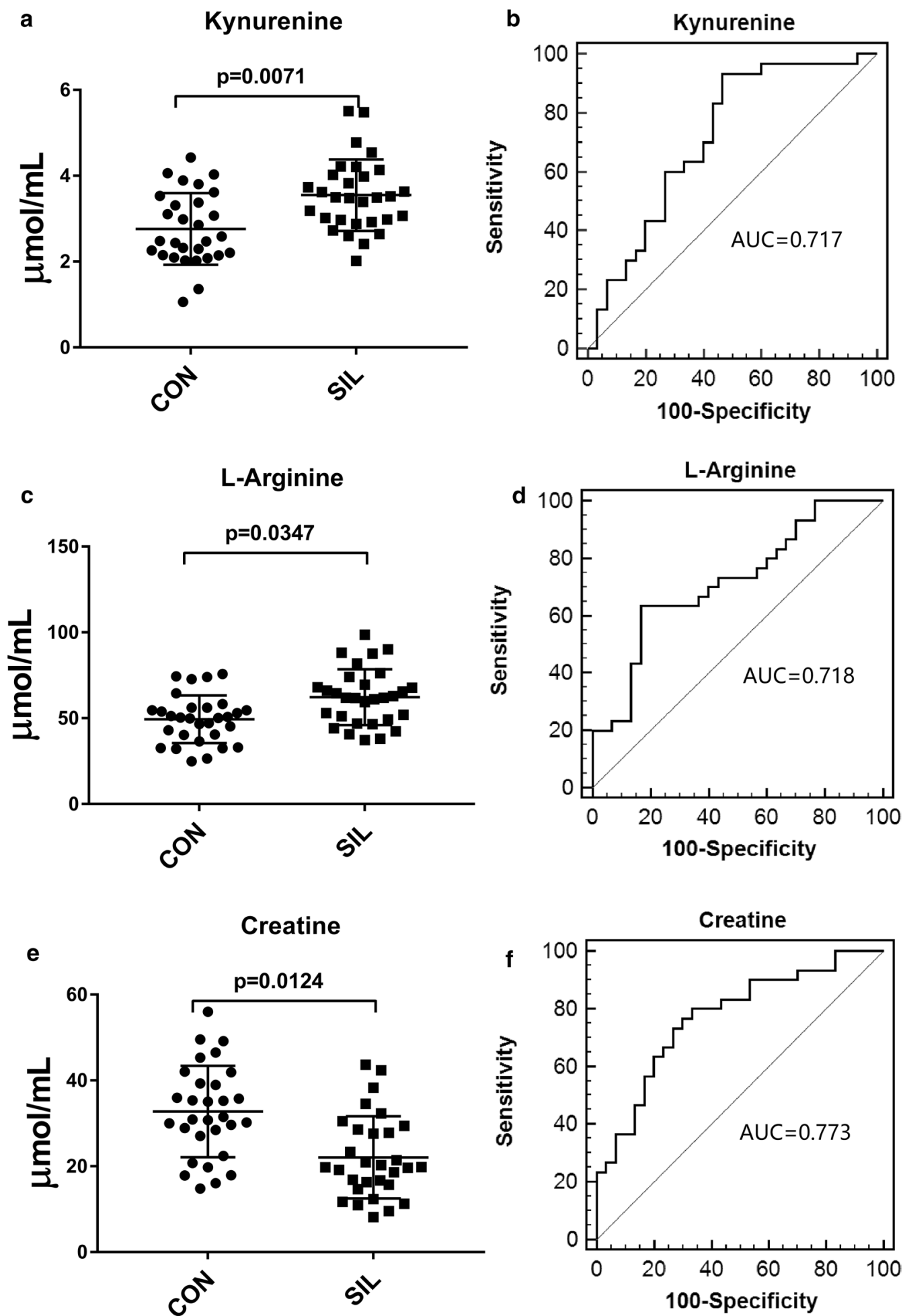

Fig. 4 Comparison of the abundance of distinct metabolic features (DMFs) in silicosis (SIL) and healthy control (CON) groups after targeted metabolomics validation and ROC analyses. $P<0.05$ indicated statistical significance. a Abundance of kynurenine. $\mathbf{b}$ ROC curve of kynurenine. $\mathbf{c}$ Abundance of $L$-arginine. $\mathbf{d}$ ROC curve of $L$-arginine. e Abundance of creatine. $\mathbf{f}$ ROC curve of creatine 
Table 2 Plasma concentrations of kynurenine, L-arginine and creatine in the patients with various stages of silicosis

\begin{tabular}{lllll}
\hline & Stage I & Stage II & Stage III & $P$ value \\
\hline $\mathrm{N}$ & 9 & 8 & 13 & \\
Kynurenine $(\mu \mathrm{mol} / \mathrm{mL})$ & $3.1 \pm 0.4$ & $3.1 \pm 0.7$ & $4.1 \pm 0.8^{*} \boldsymbol{\Delta}$ & 0.002 \\
L-arginine $(\mu \mathrm{mol} / \mathrm{mL})$ & $71.4 \pm 14.0$ & $58.7 \pm 19.2$ & $58.3 \pm 14.4$ & 0.137 \\
Creatine $(\mu \mathrm{mol} / \mathrm{mL})$ & $24.8 \pm 8.9$ & $22.8 \pm 10.4$ & $19.8 \pm 9.7$ & 0.480 \\
\hline
\end{tabular}

Data was presented as mean $\pm \mathrm{SD}$

${ }^{*}$ Compared with Stage I, $P$ value $<0.05 ;{ }^{\Delta}$ Compared with Stage II, $P$ value $<0.05$

In the present study, the distinct metabolic features in the plasma of the patients of silicosis compared with that of DEWs were identified to be different. Five lipid DMFs were still metabolism increased in silicosis, such as phosphatidylcholine and LysoPE. Three amino acid DMFs, L-arginine, kynurenine and creatine, could distinguish patients with silicosis from the healthy controls with ROC curve analysis. Amino acids are the basic components of proteins and the raw materials for protein synthesis, and they participate in the complex metabolic process of the body. Increased levels of some amino acids, including creatine, putrescine, spermidine, 4-hydroxyproline and proline-hydroxyproline dipeptide, were found in the fibrotic lung tissue of patients with IPF compared with normal lung tissue [17]. Collagen fibrils are the most abundant protein in the ECM, and excess collagen deposition in the ECM is associated with the key pathogenic mechanism of IPF [34]. Ornithine can also be converted to proline and hydroxyproline for collagen formation in fibrosis [18]. Silicosis has similar changes in amino acid metabolism as IPF, which may be related to the fibrotic process [19]. In the late pathogenesis of silicosis, the formation of silicotic nodules is the pathological manifestation of the production of collagen fibres and pulmonary interstitial fibrosis mediated by amino acid metabolism, which is consistent with the finding in the present study that arginine and proline metabolism was the major metabolic pathway in silicosis.

A growing number of studies have suggested that arginine methylation and asymmetric dimethylarginine metabolism may be associated with the progression of IPF [35]. One animal experiment featuring lung fibrosis showed that the direct infusion of asymmetric dimethylarginine resulted in elevated collagen deposition in mouse lungs and enhanced arginase activity [36]. $\mathrm{L}$-arginine can produce $\mathrm{L}$-ornithine under the action of arginase, and then, through ornithine aminotransferase, $\mathrm{L}$-ornithine converts into proline, which is the main component of collagen [37]. Our data has shown that the plasma level of L-arginine in silicosis was significantly higher than that in the controls and was related to the decline of pulmonary function. Kynurenine is an intermediate metabolite of tryptophan, an essential amino acid. Kynurenine has immune regulatory functions and can regulate vascular tone, which might be relevant in pulmonary hypertension [38]. Pulmonary hypertension is related to pulmonary artery stenosis resulting from mechanical compression by the lesions of central type progressive massive fibrosis in silicosis. We found that compared with that of the controls, the level of kynurenine significantly increased in silicosis. Moreover, the level of kynurenine was higher in Stage III silicosis than in Stage I or Stage II silicosis and was negatively associated with $\mathrm{FVC} \%$ predicted. This finding may partially explain the overexpression of kynurenine in severe silicosis.

Some limitations of the present study should be mentioned. First, potential enrolment bias existed in the present study and may affect the validity of the results. The study population from a single medical centre may not be fully representative of all patients with silicosis. In addition, more males were enrolled than
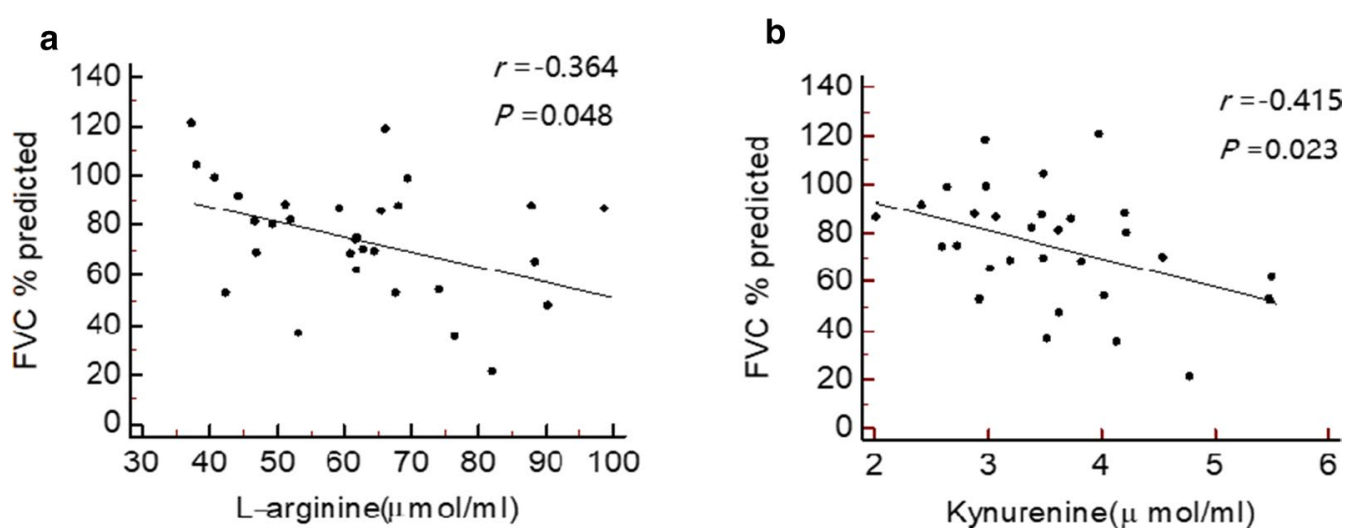

Fig. 5 Correlations between L-arginine and kynurenine and the predicted percentage of forced vital capacity (FVC\% predicted) in silicosis. a L-arginine and FVC\% predicted. b Kynurenine and FVC\% predicted 
females because they were at risk of silica dust exposure through engagement in manual labour, such as excavation and digging, polishing and buffing. Second, other lung diseases such as chronic obstructive pulmonary disease and some exogenous factors such as smoking, alcohol and medication may also lead to metabolic changes $[39,40]$. These were not included in this study. The possible effects of these factors were warranted to be assessed in further study. Third, although the plasma metabolic features of silicosis are accessible and noninvasive, they may not fully represent the metabolic process of the lungs. Further research is warranted to explore the distinct metabolism of sputum as well as bronchoalveolar lavage in patients with silicosis. Finally, given the cross-sectional design, the study did not have the power to explore the dynamic metabolites for disease progression and survival, which are clinically significant.

\section{Conclusions}

The present study provided important information regarding sphingolipid metabolism and arginine and proline metabolism in DEWs and patients with silicosis, respectively. The distinct metabolic features in the plasma of the patients of silicosis compared with that of DEWs were found to be different. In the validation metabolomics study, our results showed that L-arginine and kynurenine were correlated with the severity of silicosis, and may have a predictive role in monitoring this disease. Further study is warranted to explore the metabolic mechanisms and the possibility of intervention in or prevention of silicosis.

\begin{abstract}
Abbreviations
AUC: Area under the curve; DEW: Dust-exposed worker; DMFs: Distinct metabolic features; ECM: Extracellular matrix; FVC: Forced vital capacity; ILO: International Labour Organization; IPF: Idiopathic pulmonary fibrosis; KEGG: Kyoto Encyclopedia of Genes and Genomes; LysoPC: Lysophosphatidylcholine; LysoPE: Lysophosphatidylethanolamine; MS: Mass spectrometry; OPLS-DA: Orthogonal partial least-squares-discriminant analysis; PCA: Principal component analysis; QC: Quality control; ROC: Receiver operating characteristic; UHPLC-MS: Ultra-high performance liquid chromatography-mass spectrometry; VIP: Variable importance for projection.
\end{abstract}

\section{Supplementary Information}

The online version contains supplementary material available at https://doi. org/10.1186/s12890-021-01462-1.

Additional file 1. Additional file contains supplemental methods and supplemental Figures and Tables as referenced in the manuscript.

\section{Acknowledgements}

We thank our colleagues Shuang Li and Lili Zhang for their assistance in the study. We would also like to express our thanks to Miss Moyang Xu of the
University of Michigan Ann Arbor for editing the language and grammar of the manuscript.

\section{Authors' contributions}

CX performed all data collection, collected and processed samples, analysing the data and wrote the manuscript. NW, YF and JM were responsible for recruiting the patients. QY contributed as primary investigator and was responsible for designing the study, recruiting the patients and writing the manuscript. All authors read and approved the final manuscript.

\section{Funding}

The work was supported by Project of Beijing Science and Technology (Z181100001718118) and National Natural Science Foundation of China (81970061).

\section{Availability of data and materials}

The datasets used and/or analysed during the current study are available from the corresponding author on reasonable request.

\section{Declarations}

\section{Ethics approval and consent to participate}

All investigations were conducted in accordance with the ethical standards of Beijing Chao-Yang Hospital and the World Medical Association Declaration of Helsinki. The study was approved by the Institutional Review Board of Beijing Chao-Yang Hospital with approval number 2018-Sci-119. Written informed consent was obtained from all individuals.

\section{Consent for publication}

Not applicable.

\section{Competing interests}

The authors declare that they have no competing interests.

Received: 25 November 2020 Accepted: 9 March 2021

Published online: 17 March 2021

\section{References}

1. Leung CC, Yu IT, Chen W. Silicosis. Lancet. 2012;379:2008-18.

2. Blanc PD, Annesi-Maesano I, Balmes JR, et al. The occupational burden of nonmalignant respiratory diseases: an official American Thoracic Society and European Respiratory Society Statement. Am J Respir Crit Care Med. 2019;199:1312-34.

3. Wu N, Xue C, Yu S, et al. Artificial stone-associated silicosis in China: a prospective comparison with natural stone-associated silicosis. Respirology. 2020;25(5):518-24.

4. Chen W, Liu Y, Wang H, et al. Long-term exposure to silica dust and risk of total and cause-specific mortality in Chinese workers: a cohort study. PLoS Med. 2012;9:e1001206.

5. Castranova V, Vallyathan V. Silicosis and coal workers' pneumoconiosis. Environ Health Perspect. 2000;108(Suppl 4):675-84.

6. Pollard KM. Silica, silicosis, and autoimmunity. Front Immunol. 2016;7:97.

7. Liu $\mathrm{H}$, Cheng $Y$, Yang J, et al. BBC3 in macrophages promoted pulmonary fibrosis development through inducing autophagy during silicosis. Cell Death Dis. 2017:8(3):e2657.

8. Nardi J, Nascimento S, Goethel G, et al. Inflammatory and oxidative stress parameters as potential early biomarkers for silicosis. Clin Chim Acta. 2018;484:305-13.

9. Miao R, Ding B, Zhang Y, et al. Proteomic profiling change during the early development of silicosis disease. J Thorac Dis. 2016;8(3):329-41.

10. Zhu Y, Yao J, Duan Y, et al. Protein expression profile in rat silicosis model reveals upregulation of PTPN2 and its inhibitory effect on epithelialmesenchymal transition by dephosphorylation of STAT3. Int J Mol Sci. 2020;21(4):1189.

11. Bo C, Geng X, Zhang J, et al. Comparative proteomic analysis of silica induced pulmonary fibrosis in rats based on tandem mass tag (TMT) quantitation technology. PLoS ONE. 2020;15(10):e0241310. 
12. Xue C, Wu N, Li X, et al. Serum concentrations of Krebs von den Lungen-6, surfactant protein D, and matrix metalloproteinase-2 as diagnostic biomarkers in patients with asbestosis and silicosis: a case-control study. BMC Pulm Med. 2017;17(1):144.

13. Aksu N, Samadi A, Yalcinkaya A, et al. Evaluation of oxysterol levels of patients with silicosis by LC-MS/MS method. Mol Cell Biochem. 2020;467(1-2):117-25.

14. Johnson CH, Ivanisevic J, Siuzdak G. Metabolomics: beyond biomarkers and towards mechanisms. Nat Rev Mol Cell Biol. 2016;17:451-9.

15. Trujillo E, Davis C, Milner J. Nutrigenomics, proteomics, metabolomics, and the practice of dietetics. J Am Diet Assoc. 2006;106:403-13.

16. Kottmann RM, Kulkarni AA, Smolnycki KA, et al. Lactic acid is elevated in idiopathic pulmonary fibrosis and induces myofibroblast differentiation via $\mathrm{pH}$-dependent activation of transforming growth factor-beta. Am J Respir Crit Care Med. 2012;186:740-51.

17. Kang YP, Lee SB, Lee JM, et al. Metabolic profiling regarding pathogenesis of idiopathic pulmonary fibrosis. J Proteome Res. 2016;15:1717-24.

18. Zhao YD, Yin L, Archer S, et al. Metabolic heterogeneity of idiopathic pulmonary fibrosis: a metabolomic study. BMJ Open Respir Res. 2017;4(1):e000183.

19. Mossman BT, Churg A. Mechanisms in the pathogenesis of asbestosis and silicosis. Am J Respir Crit Care Med. 1998;157:1666-80.

20. International Labour Office. International Classification of Radiographs of Pneumoconiosis, revised. Occupational Safety and Health Series. 2011;22:Rev 2011

21. Zhao YY, Cheng XL, Lin RC. Lipidomics applications for discovering biomarkers of diseases in clinical chemistry. Int Rev Cell Mol Biol. 2014;313:1-26.

22. Zehethofer N, Bermbach S, Hagner S, et al. Lipid analysis of airway epithelial cells for studying respiratory diseases. Chromatographia. 2015;78(5-6):403-13.

23. Farooqui AA. Lipid mediators in the neural cell nucleus: their metabolism, signaling, and association with neurological disorders. Neuroscientist. 2009;15(4):392-407.

24. Yang Y, Uhlig S. The role of sphingolipids in respiratory disease. Ther Adv Respir Dis. 2011;5(5):325-44.

25. Teichgraber $\vee$, et al. Ceramide accumulation mediates inflammation, cell death and infection susceptibility in cystic fibrosis. Nat Med. 2008;14(4):382-91.

26. Nixon GF. Sphingolipids in inflammation: pathological implications and potential therapeutic targets. Br J Pharmacol. 2009;158(4):982-93.

27. Yan F, Wen Z, Wang R, et al. Identification of the lipid biomarkers from plasma in idiopathic pulmonary fibrosis by Lipidomics. BMC Pulm Med. 2017;17(1):174
28. Tager AM, Lacamera P, Shea BS, et al. The lysophosphatidic acid receptor LPA1 links pulmonary fibrosis to lung injury by mediating fibroblast recruitment and vascular leak. Nat Med. 2007;14(1):45-54.

29. Rindlisbacher B, Schmid C, Geiser T, et al. Serum metabolic profiling identified a distinct metabolic signature in patients with idiopathic pulmonary fibrosis - a potential biomarker role for LysoPC. Respir Res. 2018;19(1):7.

30. Ringseis R, Keller J, Eder K. Mechanisms underlying the anti-wasting effect of I-carnitine supplementation under pathologic conditions: evidence from experimental and clinical studies. Eur J Nutr. 2013;52(5):1421-42.

31. Saborano R, Wongpinyochit T, Totten JD, et al. Metabolic reprogramming of macrophages exposed to Silk, poly(lactic-co-glycolic acid), and silica nanoparticles. Adv Healthc Mater. 2017;6(14):1601240.

32. Peruzzi C, Nascimento S, Gauer B, et al. Inflammatory and oxidative stress biomarkers at protein and molecular levels in workers occupationally exposed to crystalline silica. Environ Sci Pollut Res Int. 2019;26(2):1394-405.

33. Anlar HG, Bacanli M, Iritaş S, et al. Effects of occupational silica exposure on oxidative stress and immune system parameters in ceramic workers in Turkey. J Toxicol Environ Health Part A. 2017;80:688-96.

34. Selman M, Thannickal VJ, Pardo A, et al. Idiopathic pulmonary fibrosis: pathogenesis and therapeutic approaches. Drugs. 2004;64(4):405-30.

35. Martinez FJ, Safrin S, Weycker D, et al. The clinical course of patients with idiopathic pulmonary fibrosis. Ann Intern Med. 2005;142(12 Pt 1):963-7.

36. Kitowska K, Zakrzewicz D, Konigshoff M, et al. Functional role and speciesspecific contribution of arginases in pulmonary fibrosis. Am J Physiol Lung Cell Mol Physiol. 2008;294(1):L34-45.

37. Maarsingh $H$, Pera T, Meurs H. Arginase and pulmonary diseases. Naunyn Schmiedebergs Arch Pharmacol. 2008;378(2):171-84.

38. Nagy BM, Nagaraj C, Meinitzer A, et al. Importance of Kynurenine in pulmonary hypertension. Am J Physiol Lung Cell Mol Physiol. 2017;313(5):L741-751.

39. Ran N, Pang Z, Gu Y, Pan H, et al. An updated overview of metabolomic profile changes in chronic obstructive pulmonary disease. Metabolites. 2019;9(6):111

40. Chen Q, Ruba SD, Ma Y, et al. Serum Metabolite biomarkers discriminate healthy smokers from COPD smokers. PLoS ONE. 2015;10(12):e0143937.

\section{Publisher's Note}

Springer Nature remains neutral with regard to jurisdictional claims in published maps and institutional affiliations.
Ready to submit your research? Choose BMC and benefit from:

- fast, convenient online submission

- thorough peer review by experienced researchers in your field

- rapid publication on acceptance

- support for research data, including large and complex data types

- gold Open Access which fosters wider collaboration and increased citations

- maximum visibility for your research: over $100 \mathrm{M}$ website views per year

At BMC, research is always in progress.

Learn more biomedcentral.com/submissions 